\title{
Periodontal bacteria in different sampling sites of pericoronitis patients
}

\author{
Ari Rajasuo $^{1}$, Virpi Laine ${ }^{2}$, Kirsti Kari ${ }^{3}$, Anna Pyhäjärvi ${ }^{3}$, Jukka H. Meurman ${ }^{3,4}$ \\ ${ }^{1}$ Department of Oral and Maxillofacial Diseases, Mikkeli Central Hospital, Mikkeli, Finland \\ ${ }^{2}$ Mikkeli Region Health Centre, Mikkeli, Finland \\ ${ }^{3}$ Institute of Dentistry, University of Helsinki, Helsinki, Finland \\ ${ }^{4}$ Department of Oral and Maxillofacial Surgery, Helsinki University Central Hospital, Helsinki, Finland \\ Email: ari.rajasuo@esshp.fi, ari.rajasuo@,fimnet.fi
}

Received 12 January 2012; revised 7 March 2012; accepted 15 March 2012

\begin{abstract}
The study investigated bacteria sampled from the mandibular third molar pericoronitis with the hypothesis that the same species also can be detected in several sites of the oral cavity. Subgingival plaque samples were taken from 41 patients (mean age $23.2 \pm$ 4.2 years), from the third molar pericoronal pocket and from 3 adjacent gingival pockets, from the tongue and saliva. Aggregatibacter actinomycetemcomitans (A.a.), Porphyromonas gingivalis (P.g.), Tannerella forsythia (T.f.), Prevotella intermedia (P.i.), Prevotella nigrescens (P.n.), and Treponema denticola (T.d.) were analyzed by qualitative PCR method. All the species analyzed were found in the pericoronitis samples in the following frequency: T.f. $73 \%$, T.d. 68\%, P.i. + P.n. 35\%, A.a. 5\%, and P.g. 5\%. Saliva and tongue samples were also highly positive with $T$.f. (both $76 \%$ ) and T.d. (saliva $57 \%$, tongue $76 \%$ ) and better sources for A.a. (saliva $8 \%$, tongue $14 \%$ ), and P.g. (saliva $14 \%$, tongue $8 \%$ ) than the pericoronitis pocket. The gingival pocket samples were less frequently positive the further the site in question was from the pericoronitis site. Contrary to earlier observations $T$.f. was frequently defected in pericoronitis sites. The results emphasize the importance of pericoronitis as a focus of infection.
\end{abstract}

Keywords: Molar; Third; Pericoronitis; Periodontitis; Bacteria; Saliva

\section{INTRODUCTION}

Predominant bacterial species of pericoronitis of erupting mandibular third molars are Streptococcus, Actinomyces and Propionibacterium species, as well as betalactamase-producing bacteria such as Prevotella, Bacteroides, Fusobacterium, Capnocytophaga, and Staphylococcus sp. [1,2]. The microbiota of pericoronitis resem- bles that of gingivitis and periodontitis [3]. However, principal periodontal pathogens have been rarely isolated from pericoronitis samples by culturing. Porphyromonas gingivalis (P.g.) has been detected in $100 \%$ of the samples of Leung et al. [3], but only in $9.1 \%$ of the samples of Rajasuo et al. [4] and in $20 \%$ of healthy third-molar pericoronal pockets by Mombelli et al. [5]. Aggregatibacter actinomycetemcomitans (A.a.) has been detected in $9.1 \%$ [4], in $17 \%-40 \%$ [5] and in $10.8 \%$ [6] of the samples, respectively. Tannerella forsythia (T.f.) has not been detected in pericoronitis samples.

Periodontal disease may also have systemic implications. It increases the risk of cardiovascular disease by approximately $20 \%$. The reported risk ratio for stroke due to periodontitis is even higher ranging from 1.7 to 2.9 [7]. For example, Pussinen et al. [8] showed that P.g. seropositive subjects had an odds ratio of 2.6 for future stroke. Their material comprised 173 subjects who experienced stroke during 13 years of follow-up, in comparison to multi-variably matched 6950 control subjects. The onset of atherosclerosis may already be in childhood and characteristically these patients have suffered from infections [9].

Subsequently, with this background, the eventual detection of P.g. and other periodontal bacterial species in specific oral infection sites is interesting in the focal infection-systemic disease paradigm also in young adults. Pericoronitis, in particular, is ranked as first [10] or second [11] in acute oral health problems of young adults.

Polymerase chain reaction (PCR) has been shown highly specific and sensitive for detecting A.a. and P.g. in periodontitis samples with a good level of agreement between the results of PCR and culture procedures in detecting these pathogens [12]. However, PCR has not been used for detecting periodontal pathogens in pericoronitis samples. Therefore, we set out to analyze six different periodontal bacterial species sampled from mandibular third-molar pericoronitis sites. The results 
were compared with those from samples taken from the gingival pockets of the adjacent teeth, from saliva, and from the dorsal aspect of tongue. The hypothesis was that the micro-organisms analyzed from the pericoronitis sites can be detected in several sampling sites in the oral cavity. The study paradigm was in focal infection theory with focus on pericoronitis as a substantial risk in young adults.

\section{MATERIAL AND METHODS}

\subsection{Patients and Clinical and $X$-Ray Recordings}

The study was conducted in the Mikkeli Region Health Centre, Southeastern Finland. Group of 41 patients (29 women, 12 men, mean age $23.2 \pm 4.2$ years) referred for out-patient oral surgery due to acute or subacute pericoronitis were included. The patients were generally healthy except one patient who had diabetes. The patients had not received antibiotic drug treatments during the last 30 days before the study. All earlier drug prescriptions were recorded from the patient records.

Pericoronitis infections were clinically regarded as mild or moderate and none of the patients had such general or local findings that would have called for extended treatment in the hospital.

Patient selection was based on the clinical diagnosis of acute or subacute pericoronitis of the mandibular third molar, with pus detected by probing the pericoronal pocket. The clinical findings recorded were axial temperature, submandibular lymphadenopathy (yes/no), limited mouth opening (yes/no), traumatizing effect of the ipsilateral maxillary third molar on the infection site (yes/no), and the clinician's plan for the treatment of pericoronitis which included drug prescriptions and timing for removal of the tooth. The World Health Organization DMFS-index for caries and CPITN for periodontal disease were calculated from clinical recordings. DT and FT indexes were also calculated for the third molar teeth. Gingival and pericoronal pockets were measured with a millimetre probe from the teeth 37 or 47,36 or 46,16 or 26,38 or 48 , when relevant. All the measurements were done after the bacterial samples had been taken.

Panoramic tomograms of the jaws were taken from all the patients to record the state of eruption of the mandibular third molars. The x-ray findings on the teeth were categorized as totally erupted, partly erupted or unerupted. Tooth inclination (degrees) was also measured from the radiographs by comparing the long axis of the tooth in question to the horizontal line drawn between the occlusal surfaces of the first and second molars. Vertical eruption level $(\mathrm{mm})$ of the third molar was also measured with help of the occlusal plane drawn.

\subsection{Bacterial Samples}

Bacterial samples were taken aseptically from six sites of each patient. The samples were taken from the pericoronitis pocket of the partly erupted mandibular third molar, from the distal gingival pockets of the adjacent mandibular first and second molars, from the distal gingival pocket of the maxillary first molar of the same site, from the dorsal surface of the tongue, and from whole saliva.

Gingival tissue at the sampling site was first cleaned with cotton swabs and contamination was carefully avoided by use of cotton rolls and saliva suction. A 0.8-mm-thick sterile endodontic paper-point was then inserted into the depth of the pericoronal and gingival pockets, respectively, and left in place for 30 seconds as described by Mombelli et al. [5] and Rajasuo et al. [4]. Saliva samples were collected from the bottom of the mouth using a sterile syringe, and tongue samples by scraping the dorsum of the tongue with a sterile metal spatula. All samples were placed immediately into an Eppendorff tube and kept deep-frozen $\left(-70^{\circ} \mathrm{C}\right)$ until sent for analyses in carbon dioxide ice. The samples were analyzed in the Research Laboratory of the Institute of Dentistry, University of Helsinki. PCR-method was used with specific primers as described by Wahlfors et al. [13] and Meurman et al. [14]. The periodontal bacteria Aggregatibacter actinomycetemcomitans (A.a.), Porphyromonas gingivalis (P.g.), Tannerella forsythia (T.f.), Prevotella intermedia (P.i.), Prevotella nigrescens (P.n.), and Treponema denticola (T.d.) were qualitatively (yes/no) analyzed from the samples.

\section{RESULTS}

Only three patients had axial temperature $>37^{\circ} \mathrm{C}\left(37.1^{\circ} \mathrm{C}\right.$ $\left.37.4^{\circ} \mathrm{C}\right) .19$ patients $(46 \%)$ had limited mouth opening and $14(34 \%)$ had submandibular lymphadenopathy. From 41 pericoronitis cases, in $21(51 \%)$ the third molar soft tissue was recorded to have been traumatized by the ipsilateral maxillary third molar. Antibiotics was prescribed to 39 (95\%) of the patients, mostly fenoxymethylpenicillin (36 cases). Roxithromycin was prescribed to 3 patients and metronidazole combined with one of the two other antibiotics also to 3 patients. Non-steroidal anti-inflammatory drugs was prescribed to $30(73 \%)$ patients, mostly ibuprofein. Chlorhexidine mouth rinses had been recommended to 13 cases.

None of the patients had gingival pockets $\geq 4 \mathrm{~mm}$, except some pseudopockets in the distal surface of the mandibular second molars due to deep location of the adjacent third molars. Ten subjects (24\%) had totally healthy periodontal status or had only supragingival calculus in the anterior mandibular teeth. In the rest $31 \mathrm{sub}-$ jects $(76 \%)$, the periodontal index scores were 0 (healthy) in $21 \%$ of the gingival sextants, 1 (bleeding when prob- 
ing) in $44 \%$ of the sextants, and 2 (calculus) in $35 \%$ of the sextants. Only 12 patients (29\%) had teeth other than third molars decayed, at a mean 2.2 teeth (SD 1.7). Correspondingly, 32 patients $(78 \%)$ had teeth other than third molars filled, at a mean 4.4 teeth (SD 3.8).

All the mandibular third molars with pericoronitis were partially erupted, 32 out of 41 (78\%) occlusal surfaces had not more than two cusps visible. No caries or dental restorations were found in these teeth. Pericoronal pockets were on an average $8.0 \pm 1.6 \mathrm{~mm}$ deep. Of the teeth $81 \%$ were either vertical or slightly distally $\left(\leq 5^{\circ}\right)$ or mesially $\left(\leq 10^{\circ}\right)$ inclined, while the rest of the third molars were mesially inclined more than $10^{\circ}$. Of the third molars $81 \%$ were located above or near the occlusal plane while $19 \%$ were $3 \mathrm{~mm}$ or more deeply located with respect to the occlusal plane. For details, see Table 1.

The bacterial samples of four patients were excluded from the study because of unsatisfactory transport to the laboratory. All the studied periodontal bacterial species were detected in all the sample sites. The number of positive samples and the number of patients positive for each species are given in the Table 2. Periodontal bacteria T.f. and T.d. were the most common species detected in all the sample sites. Their occurrence was at the same level in the pericoronitis pocket samples, in the dorsum of tongue, and in saliva samples. A.a. and P.g. were most commonly detected in saliva and tongue samples. The other gingival pocket samples were less often positive for the bacteria the further the site sampled was from the third molar periocoronitis pocket in question.

\section{DISCUSSION}

Our results indirectly showed that bacteria from mandibular third molars with pericoronitis spread in the mouth. The patients were young adults and their dental and periodontal health was good. The only oral infection focus recorded was the pericoronitis. Hence we may suppose that the periodontal bacteria detected outside the pericoronitis sites may indeed have originated from those foci to the other parts of the oral cavity. However, to verify this more accurate molecular methods are needed than used in the present study. Surprisingly, saliva and dorsum of the tongue appeared the best sampling sites for the detection of periodontal bacteria in pericoronitis patients.

We have earlier shown $[4,15]$ that third molar pericoronitis sites harbour the same bacterial species as are detected in tonsillitis and periodontitis. Furthermore, our observation [16] on Finnish conscripts in the early 1990s showed that diagnosing third molar pericoronitis presented an odds ratio up to 6.8 (with $95 \%$ confidence interval 3.0 to 15.0 ) for getting a simultaneous upper respiratory tract infection. All these data emphasize the importance of third molar pericoronitis as a focus of infection.

Table 1. Position of the mandibular third molar and depth of the follicular pocket sampled $(n=41)$.

\begin{tabular}{|c|c|c|c|c|c|c|c|c|}
\hline \multicolumn{3}{|c|}{ Depth of the pericoronal pocket } & \multicolumn{3}{|c|}{ Eruption level $(\mathrm{mm})$, under $(-)$ or above $(+)$ the occlusal plane } & \multicolumn{3}{|c|}{ Inclination } \\
\hline $4-6 \mathrm{~mm}$ & $7-9 \mathrm{~mm}$ & $10-12 \mathrm{~mm}$ & $-2--5 \mathrm{~mm}$ & $\pm 1 \mathrm{~mm}$ & $+2-+3 \mathrm{~mm}$ & Distal & Vertical $\left( \pm 5^{\circ}\right)$ & Mesial \\
\hline $22 \%$ & $56 \%$ & $22 \%$ & $34 \%$ & $44 \%$ & $22 \%$ & $3 \%$ & $50 \%$ & $47 \%$ \\
\hline
\end{tabular}

Table 2. Number and percent of positive samples of the bacterial species detected and number and percent of patients positive to each species. In total of 37 patients were analyzed.

\begin{tabular}{|c|c|c|c|c|c|}
\hline Sample site & A.a. & P.g. & P.i. + P.n. & T.f. & T.d. \\
\hline \multicolumn{6}{|l|}{ Pericoronal pocket } \\
\hline of the lower 3rd molar & $2(5.4 \%)$ & $2(5.4 \%)$ & $13(35.1 \%)$ & $27(73.0 \%)$ & $25(67.6 \%)$ \\
\hline \multicolumn{6}{|l|}{ Gingival pocket } \\
\hline of the lower 1st molar & $2(5.4 \%)$ & $1(2.7 \%)$ & $9(24.3 \%)$ & $18(48.6 \%)$ & $14(37.8 \%)$ \\
\hline of the lower 2nd molar & $3(8.1 \%)$ & $2(5.4 \%)$ & $12(32.4 \%)$ & $17(45.9 \%)$ & $15(40.5 \%)$ \\
\hline of the upper 1st molar & $2(5.4 \%)$ & $2(5.4 \%)$ & $9(24.3 \%)$ & $17(45.9 \%)$ & $10(27.0 \%)$ \\
\hline Tongue & $5(13.5 \%)$ & $3(8.1 \%)$ & $14(37.8 \%)$ & $28(75.7 \%)$ & $28(75.7 \%)$ \\
\hline Saliva & $3(8.1 \%)$ & $5(13.5 \%)$ & $20(54.1 \%)$ & $28(75.7 \%)$ & $21(56.8 \%)$ \\
\hline Total no. of positive samples & $17(7.7 \%)$ & $15(6.8 \%)$ & $77(34.7 \%)$ & $135(60.8 \%)$ & $113(50.9 \%)$ \\
\hline No. of patients positive & $9(24.3 \%)$ & $8(21.6 \%)$ & $23(62.2 \%)$ & $36(97.3 \%)$ & $32(86.5 \%)$ \\
\hline
\end{tabular}


Friedlander et al. [17] recently investigated panoramic jaw X-rays of subjects with ultrasonically verified carotid artery atheromas and observed a significantly more frequently signs of oral infections among those with vs without carotid atheromas. In their material, however, no difference was seen in this respect with subjects with or without pericoronitis but the number of observations was low. Söder et al. [18] have also shown that long-term periodontitis increases the risk for sub-clinical atherosclerosis. Hence, it remains to be shown whether or not having pericoronitis, which is common infection in young adulthood, increases the risk for other systemic diseases such as the development of the atherosclerosis.

The weaknesses of study were the low number of patients and the fact that we did not verify the origin of the bacteria analyzed by using further molecular techniques, for example. At the time of investigation quantitative real-time PCR was not available in our laboratory. Thus more studies are called for final conclusion. Nevertheless to our knowledge the present study was the first of its kind and thus provides basis for further hypothesis generation.

In conclusion, we have shown that periodontal bacteria, which in our patients might have originated from the mandibular third molar pericoronitis sites, could be detected from samples outside the actual infection focus. The result supports the concept of pericoronitis as an infection with potential further consequences.

\section{ACKNOWLEDGEMENTS}

Professors Mariano Sanz and David Herrera from the Complutense University of Madrid, Spain, are thanked for their advice when planning this investigation.

The study was supported by grants from the Mikkeli Central Hospital and the Helsinki University Central Hospital, Finland.

Preliminary results of the same material were published in the poster session of the annual congress of IADR (International Association for Dental Research), in 2010, Barcelona, Spain. The abstract of the presentation can be found in http://iadr.confex.com/iadr/2010barce/webprogram/, Paper135301.html.

The study was approved by the Ethical committee of Mikkeli Central Hospital and Mikkeli Region Health Centre. Informed consent was obtained from the patients.

\section{REFERENCES}

[1] Gutiérrez Pérez, J.L. (2004) Third molar infections. Medicina Oral Patologia Oral y Cirugia Bucal, 9, 120-125.

[2] Sixou, J.-L., Magaud, C., Jolivet-Gougeon, A., et al. (2003) Evaluation of the mandibular third molar pericoronitis flora and its susceptibility to different antibiotics prescribed in France. Journal of Clinical Microbiology, 41, 5794-5797. doi:10.1128/JCM.41.12.5794-5797.2003
[3] Leung, W.K., Theilade, E., Comfort, M.B., et al. (1993) Microbiology of the pericoronal pouch in mandibular third molar pericoronitis. Oral Microbiology and Immunology, $\mathbf{8}, 306-312$. doi:10.1111/j.1399-302X.1993.tb00579.x

[4] Rajasuo, A., Jousimies-Somer, H., Savolainen, S., et al. (1996) Bacteriologic findings in tonsillitis and pericoronitis. Clinical Infectious Diseases, 23, 51-60. doi:10.1093/clinids/23.1.51

[5] Mombelli, A., Buser, D., Lang, N.P., et al. (1990) Suspected periodontopathogens in erupting third molar sites of periodontally healthy individuals. Journal of Clinical Periodontology, 17, 48-54. doi:10.1111/j.1600-051X.1990.tb01047.x

[6] Peltroche-Llacsahuanga, H., Reichhart, E., Schmitt, W., et al. (2000) Investigation of infectious organisms causing pericoronitis of the mandibular third molar. Journal of Oral and Maxillofacial Surgery, 58, 611-616. doi: $10.1053 /$ jo. 2000.6200

[7] Meurman, J.H., Sanz, M. and Janket, S.-J. (2004) Oral health, atherosclerosis and cardiovascular disease. Critical Reviews in Oral Biology \& Medicine, 15, 403-413. doi: $10.1177 / 154411130401500606$

[8] Pussinen, P.J., Alfthan, G., Rissanen, H., et al. (2004) Antibodies to periodontal pathogens and stroke risk. Stroke, 35, 2020-2023. doi:10.1161/01.STR.0000136148.29490.fe

[9] Jönsson, G., Truedsson, L., Sturfelt, G., et al. (2005) Hereditary C2 deficiency in Sweden: Frequent occurrence of invasive infection, atherosclerosis, and rheumatic disease. Medicine, 84, 23-34.

[10] Guralnick, W. (1984) Third molar surgery. British Dental Journal, 156, 389-394. doi:10.1038/sj.bdj.4805380

[11] Ludwick, W.E., Gendron, E.G., Pogas, J.A., et al. (1974) Dental emergencies occurring among Navy-Marine personnel serving in Vietnam. Military Medicine, 139, 121123.

[12] Lau, L., Sanz, M., Herrera, D., et al. (2004) Quantitative real-time polymerase chain reaction versus culture: A comparison between two methods for the detection and quantification of Actinobacillus actinomycetemcomitans, Porphyromonas gingivalis and Tannerella forsythensis in subgingival plaque samples. Journal of Clinical Periodontology, 31, 1061-1069. doi:10.1111/j.1600-051X.2004.00616.x

[13] Wahlfors, J., Meurman, J.H., Väisänen, P., et al. (1995) Simultaneous detection of Actinobacillus actinomycetemcomitans and Porphyromonas gingivalis by a rapid PCR method. Journal of Dental Research, 74, 17961801. doi:10.1177/00220345950740111301

[14] Meurman, J.H., Wahlfors, J., Korhonen, A., et al. (1997) Identification of Bacteroides forsythus in subgingival dental plaque with the aid of a rapid PCR method. Journal of Dental Research, 76, 1376-1380. doi:10.1177/00220345970760070701

[15] Rajasuo, A., Sihvonen, O.J., Peltola, M., et al. (2007) Periodontal pathogens in erupting third molars of 
periodontally healthy subjects. International Journal of Oral and Maxillofacial Surgery, 36, 818-821. doi:10.1016/j.ijom.2007.05.001

[16] Meurman, J.H., Rajasuo, A., Murtomaa, H., et al. (1995) Respiratory tract infections and concomitant pericoronitis of the wisdom teeth. British Medical Journal, 310, 834836. doi:10.1136/bmj.310.6983.834

[17] Friedlander, A.H., Sung, E.C., Chung, E.M., et al. (2010) Radiographic quantification of chronic dental infection and its relationship to the atherosclerotic process in the carotid arteries. Oral Surgery, Oral Medicine, Oral Pathology, Oral Radiology \& Endodontics, 109, 615-621. doi:10.1016/j.tripleo.2009.10.036

[18] Söder, P.O., Söder, B., Nowak, J., et al. (2005) Early carotid atherosclerosis in subjects with periodontal diseases. Stroke, 36, 1195-1200.

doi:10.1161/01.STR.0000165916.90593.cb 